\title{
A Prolog-Based Tutor for Multi-column Subtraction with Multiple Algorithms Support (Software Demonstration)
}

\author{
Claus Zinn \\ Department of Computer Science, University of Konstanz \\ Funded by the DFG (ZI 1322/2/1) \\ claus.zinn@uni-konstanz.de
}

\begin{abstract}
We present an intelligent tutoring system capable of analysing learner input across multiple reference models. Its main component, the diagnoser, is build upon logic programming techniques. It uses a novel variant of Shapiro's algorithmic debugging method, whose scientific aspect is described in our full technical contribution to KI-13 [3]. In this poster, we show the use of the diagnoser in the overall tutoring system.
\end{abstract}

\section{Introduction}

Intelligent systems appear intelligent because they are designed to perform in well-defined contexts. This also holds for intelligent tutoring systems (ITSs), where the diagnosis of learner behaviour is best possible in domains that tend to be highly structured, and for which problem solving strategies can be easily represented and executed. It is clear that ITS designers must define the system's domain of instruction, and thus the amount of expertise that it requires. But all too often, the designers also expect learners to solve tasks in the chosen domain by following a single, prescribed algorithm. The actions that learners can perform are further constrained by the design of the system's graphical user interface (GUI) and the design of the interaction. Often, learners are forced to submit their solution in a piece-meal fashion, leaving little room for learners to explore, and deviate from, the solution space on their own.

All constraints have rather practical than pedagogical reasons. They reduce the cost of authoring, and keep manageable the computational complexity of the diagnosis engine. In addition, if learners were allowed to exhibit multiple problem solving strategies, then the system must be capable of identifying the strategy learners are most likely following. With learner input being often erroneous, this task is not trivial.

Our method for the cognitive diagnosis of input is based upon an innovative use of Shapiro's algorithmic debugging technique [1], whose adaptation is described in [2]. Our technical contribution to KI-2013 describes how we extended our method to take into account multiple reference models for the analysis of learner input [3]. As a result, we are able to loosen the tight leash between tutoring system and learner. In this system demonstration paper, we describe the practical use of our method in a prototypical intelligent tutoring system. 


\section{The Domain of Instruction}

Our chosen domain of instruction is multi-column subtraction, an area well studied in the intelligent tutoring community. There are several algorithms.

In the Austrian method, sums are processed column by column, from right to left. If the subtrahend S of the current column is greater than the column's minuend $\mathrm{M}$, then $\mathrm{M}$ is increased by 10 (borrowing) before the difference $\mathrm{M}-\mathrm{S}$ is taken. To compensate, the $\mathbf{S}$ in the column left to the current one is increased by one (payback). The trade-first variant of the Austrian method performs the same steps than the Austrian method, but in a different order. First, all payback and borrowing operations are executed, then all differences are taken. The decomposition method realizes the payback operation by decrementing minuends rather than incrementing subtrahends. The left-to-right method processes sums in the opposite direction; also, payback operations are performed on result rather than minuend or subtrahend cells. - All methods have been implemented in (SWI-)Prolog, see http://www.swi-prolog.org/.

\section{The Look and Feel of the Tutoring System}

Figure 1 depicts the GUI of our subtraction tutor. It has four main areas.
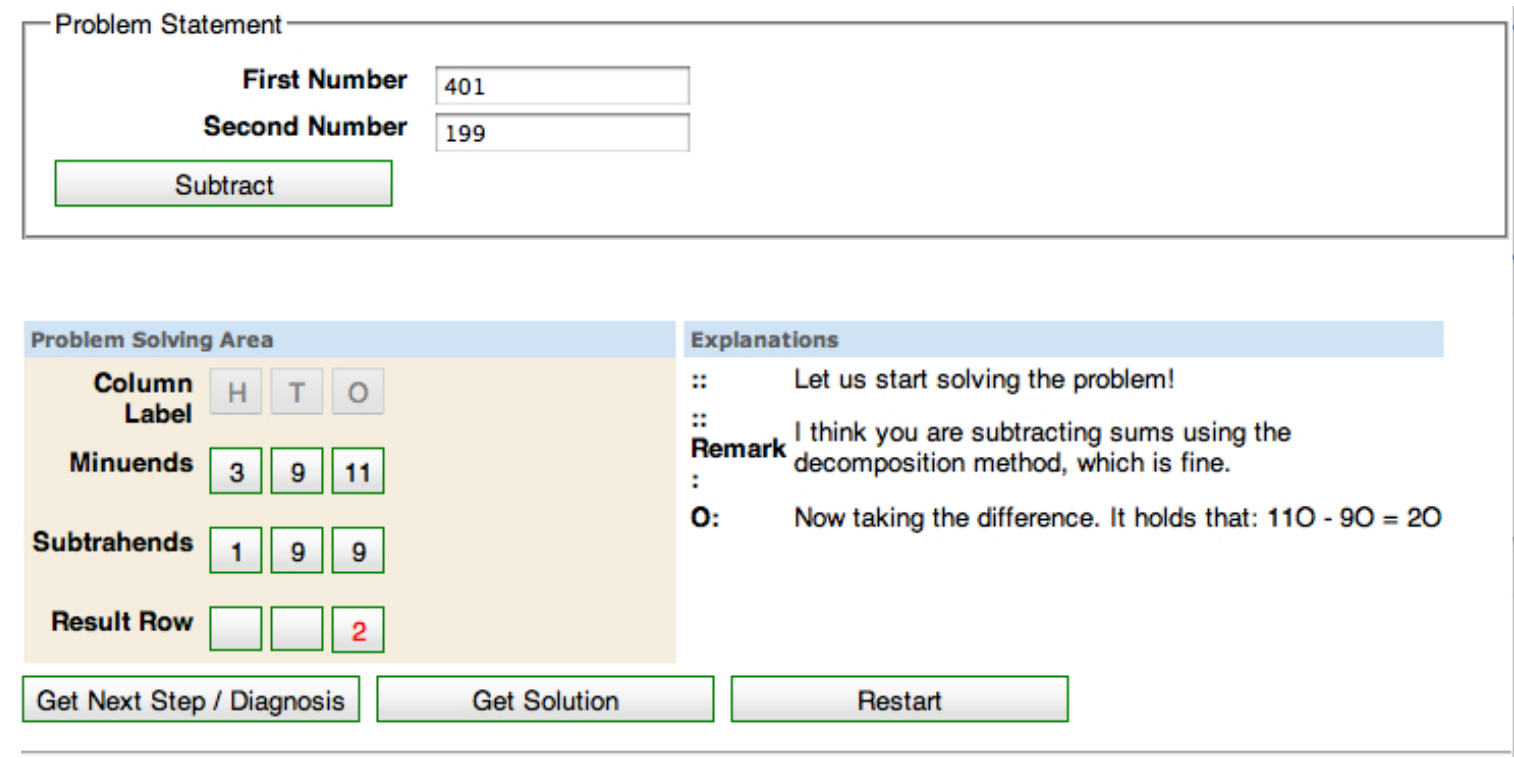

Fig. 1. The Front-End of the Tutoring System

The problem statement area (top) allows users to define their own subtraction problems. Unlike other systems, there are no predefined problems that learners must choose from. When the user enters two arbitrary integers and presses "Subtract", the problem solving area is automatically generated, with the task to subtract the smaller from the larger number. 
The problem solving area (middle left) display the subtraction problem as a matrix of four rows. Each column has a label ("ones" $(\mathrm{O})$, "tens" (T), "hundreds" $(\mathrm{H})$ etc.) as well as a minuend, subtrahend, and result cell. Learners can interact with all but the label cells, and in arbitrary order. A click on a minuend or subtrahend cell (resp. result cell) opens a number pane with numbers ranging from 0 to 19 (resp. 0 to 9 ); the learner's selection is entered in the cell.

In the explanation area (middle right), learners receive feedback when asking for next-step help, or the diagnosis of their input. All feedback is directly derived from our algorithmic debugger, which determines the first irreducible disagreement between the tutor's expert subtraction method (the best-ranked method, which the system believes the learner is following) and observed learner behaviour [2,3]. System feedback is verbalised using template-based natural language generation. Feedback also involves filling or modifying cells in the subtraction matrix; those are marked red.

In the command area, learners can request - at any time during problem solving - help or diagnosis, the entire solution to a given problem, or a restart.

The GUI is designed not to favour any particular subtraction method. Learners can perform actions following any of the four subtraction methods, or erroneous variants thereof. Moreover, learners can click on any of the interaction elements at any time; system feedback is only given when learners request it.

\section{Technical Architecture}

The client-server architecture of our system follows a Model-View-Controller (MVC) design pattern, see Fig. 2. There is, thus, a clear separation of knowledge sources, which supports the division of labour among Prolog and GUI developers.

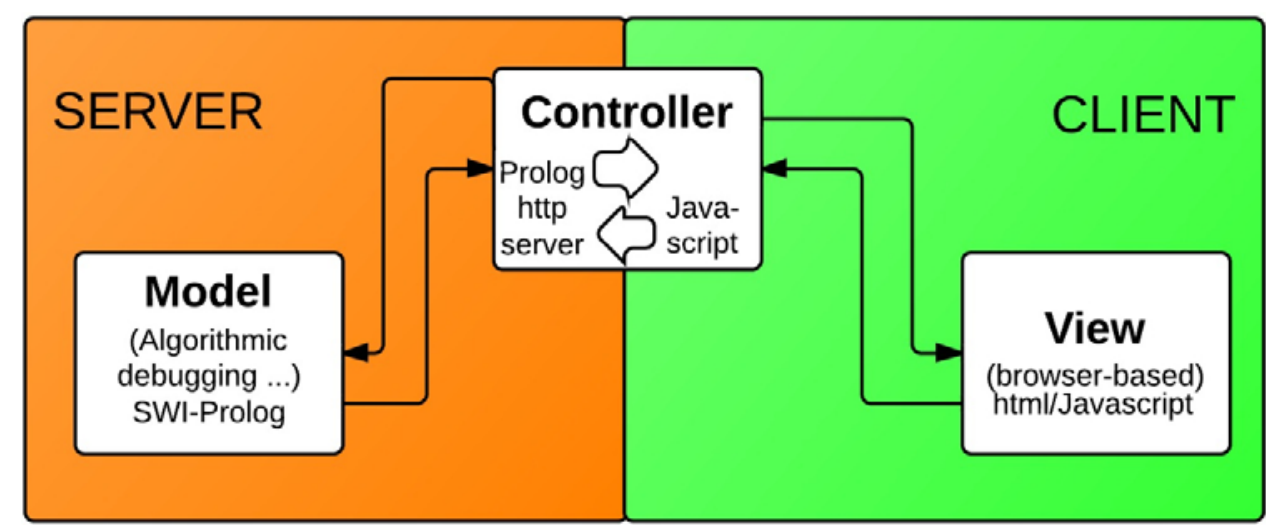

Fig. 2. The Technical Architecture of the Tutoring System

The model, hosted on the server, encapsulates an application's business logic or state. In our system, the model is entirely implemented in Prolog. It contains: (i) four modules, each containing one algorithm for multi-column subtraction; (ii) a domain-independent diagnosis module that encodes algorithmic debugging, 
including the ranking method that identifies the strategy the learner is most likely following [3]; (iii) four modules, each mechanising the Oracle for one of the subtraction algorithms; and (iv) a state handler, which stores the current subtraction task, its solution, and some other state-related information.

The view, on the client-side, represents an application's GUI. It displays a representation of the world state, allows users to perform actions to manipulate the state, and displays graphical and textual feedback. It is written in HTML and Javascript and makes use of the Dojo Toolkit http://dojotoolkit.org/.

All information flow between the model and the view is mediated by the controller. The controller is divided in a client-side (Javascript) and a serverside (Prolog) component. The client-side controller translates a GUI interaction into an HTTP request that is sent to the server-side controller, which translates it to an appropriate request to the model. When the model's answer is received, it is then mediated back from the server-side controller to the client-side controller, which in turn, instructs the view to update its display accordingly.

\section{Conclusion and Future Work}

We have presented a subtraction tutor that loosens the tight leash between system and learners. Our learners are free to follow one of four possible problem solving strategies; the tutor's GUI and interaction design restricts learners' scope of action as little as possible; and system feedback will only be delivered upon request. In [3], we show that our approach increases the diagnostic capability of the system, despite of the wider range of input that needs to be processed. The computational costs are negligible, permitting the addition of more strategies.

In the future, we would like to adapt the GUI so that school children can comfortably use it. For this, we will include teachers and learners to improve the system's usability. We also envision a system where learners can solve subtraction problems more naturally, and have started integrating a handwriting recognition engine. Ideally, we want users of touch-enabled computer devices to solve problems very close to the natural paper and pencil approach.

System Availability. The subtraction tutor is available at http://www.inf.uni-konstanz.de/ zinn/. We have also implemented single-model tutors for each of the subtraction methods. Feedback is highly welcome.

\section{References}

1. Shapiro, E.Y.: Algorithmic Program Debugging. ACM Distinguished Dissertations. MIT Press (1983); Thesis (Ph.D.) - Yale University (1982)

2. Zinn, C.: Algorithmic debugging to support cognitive diagnosis in tutoring systems. In: Bach, J., Edelkamp, S. (eds.) KI 2011. LNCS, vol. 7006, pp. 357-368. Springer, Heidelberg (2011)

3. Zinn, C.: Algorithmic debugging for intelligent tutoring: How to use multiple models and improve diagnosis. In: Timm, I.J., Thimm, M. (eds.) KI 2013. LNCS (LNAI), vol. 8077, pp. 272-283. Springer, Heidelberg (2013) 